\title{
Erratum to: The guanine nucleotide exchange factor Vav3 regulates differentiation of progenitor cells in the developing mouse retina
}

\author{
Veronika Luft • Jacqueline Reinhard • Masabumi Shibuya •
}

Klaus D. Fischer • Andreas Faissner

Published online: 12 March 2015

(C) Springer-Verlag Berlin Heidelberg 2015

\section{Erratum to: Cell Tissue Res}

DOI 10.1007/s00441-014-2050-2

In the old version of Fig. 8 the micrographs $\mathrm{k}$ and 1 were inadvertently exchanged.

As we noted no differences between the Vav3 knock-out and wild-type situation, the sense of our conclusion remains untouched and is still correct.

The quantitative analysis was performed with the correct data.

The authors apologize for this mistake.

The online version of the original article can be found at http://dx.doi.org/ 10.1007/s00441-014-2050-2.

V. Luft · J. Reinhard · A. Faissner $(\bowtie)$

Department of Cell Morphology \& Molecular Neurobiology,

Ruhr-University Bochum NDEF 05/594, Universitaetsstr.150,

D-44780 Bochum, Germany

e-mail: Andreas.Faissner@ruhr-uni-bochum.de

V. Luft

International Graduate School of Neuroscience (IGSN), FNO 1/116

Universitaetsstr.150, D-44780 Bochum, Germany

M. Shibuya

Division of Genetics, Institute of Medical Science, University of

Tokyo, 4-6-1 Shirokanedai, Minato-ku Tokyo 108-8639, Japan

\section{K. D. Fischer}

Institute of Biochemistry and Cell Biology, Otto-von-Guericke

University Magdeburg, Leipziger Straße 44,

D-39120 Magdeburg, Germany 
P6
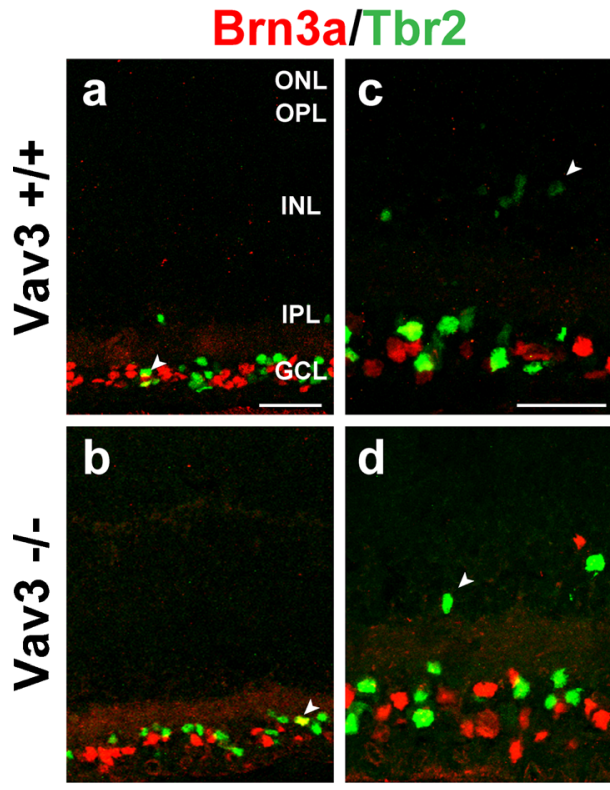

e

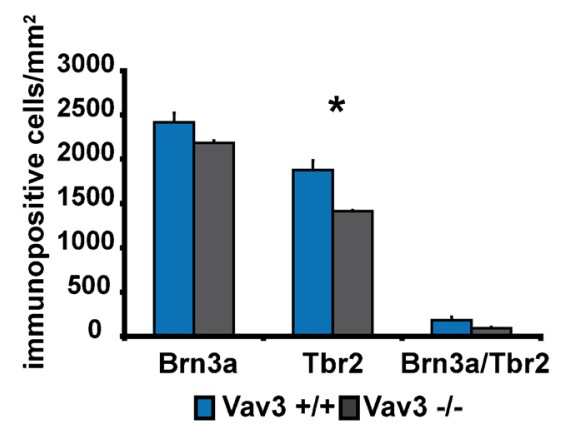

口 Vav3 +/+םVav3 -/-
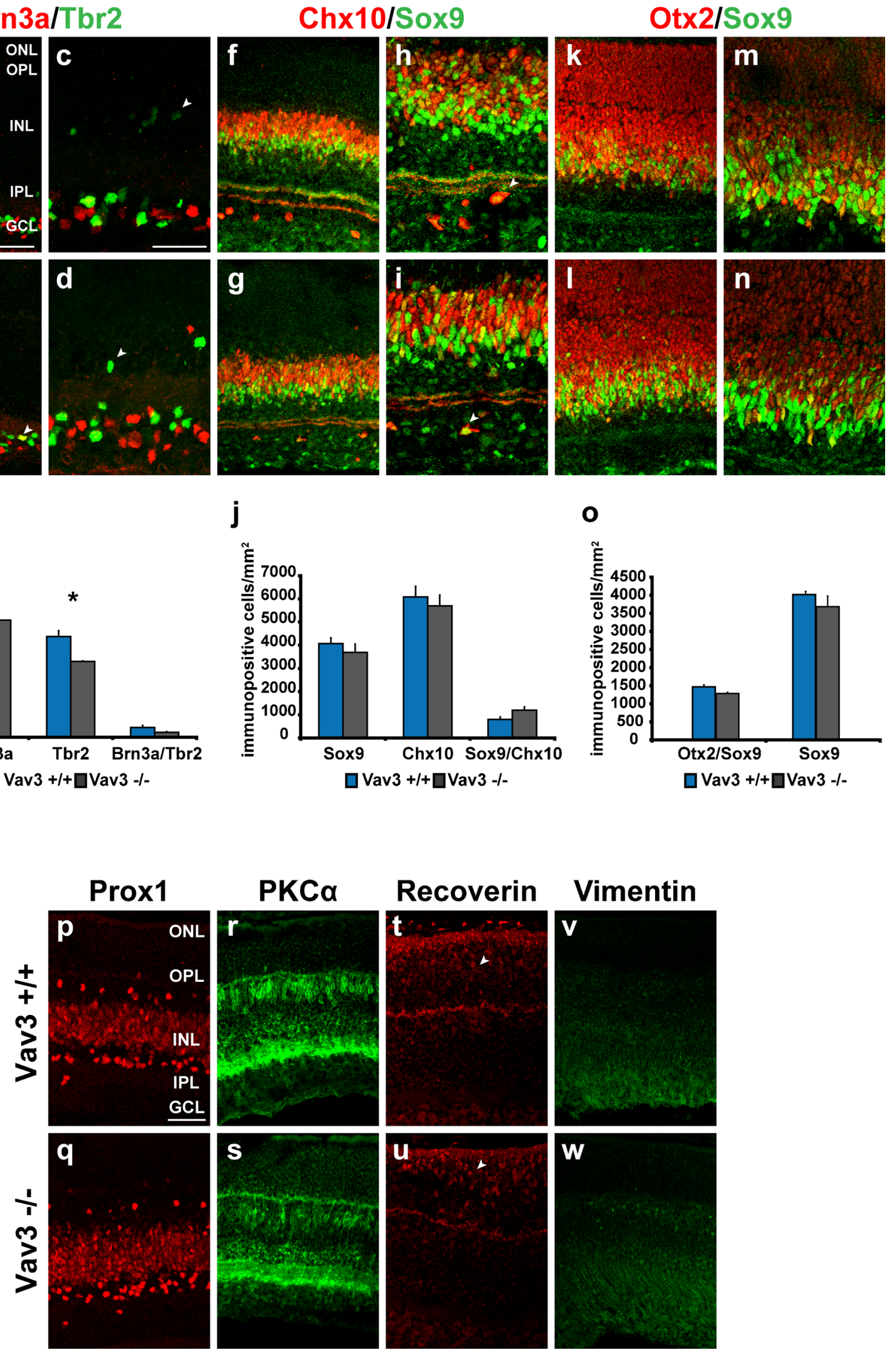

\section{Vimentin}
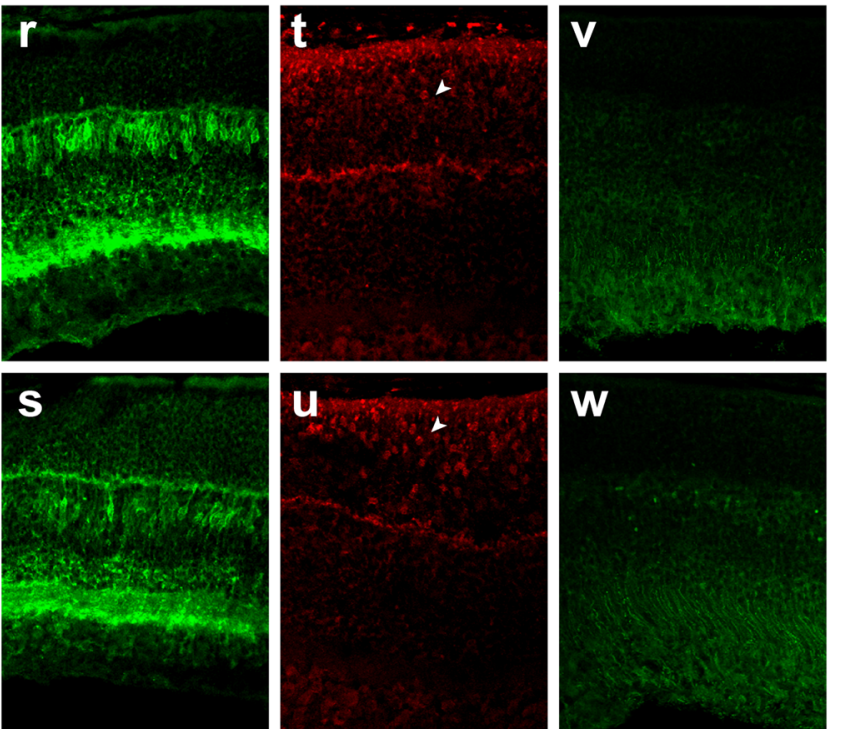
4 Fig. 8 Neuronal cell types in the postnatal Vav3 deficient retina. (a-b) Double-labeling against the RGC markers Tbr2 and Brn3a in wild-type and Vav3 -/- retinas was performed at P6. (c-d) Higher magnifications show that Brn3a-positive cells were mainly restricted to the GCL, while single Tbr2-positive cells were located in outer parts of the retina (arrowheads). These cells were not considered for quantification, because they are most likely amacrine cells. (e) Quantification revealed a comparable proportion of Brn3a-positive cells in both genotypes, while the number of Tbr2-expressing cells was significantly decreased in the Vav3 deficient retina. (f-i) Antibodies against Chx10 and Sox9 were used to label bipolar cells and Müller glia in the INL. We also detected single positive cells in the GCL, which the typical morphology of displaced amacrine cells (arrowheads). (j) Here, we found no significant differences in the number of Sox9-, Chx10- or Sox9/Chx10-double positive cells between both genotypes. (k-o) The same was true for Sox9- or Otx2/Sox9-double positive cells. (p-q) Horizontal, amacrine and bipolar cells were detected with antibodies against Prox1. The majority of these cells were localized in the center of the INL. Two additional bands of Prox1-positive cells were detectable at the apical and basal margin of the INL. (r-s) PKC $\alpha$, a marker for bipolar cells, labeled cell bodies in the INL and the synaptic terminals in the IPL. ( $t$ u) Immunohistochemical detection of recoverin-positive photoreceptor cells (arrowheads) in the ONL. (v-w) Staining against vimentin revealed Müller glia processes spanning the inner part of the retina. With these markers, we could not detect any differences between wildtype and Vav3 -/- mice in the postnatal retina. Statistical analysis was performed using the unpaired two-sided Student's $t$-test $\left({ }^{*} P \leq 0.05\right.$, $* * P \leq 0.01, * * * P \leq 0.001)$. GCL ganglion cell layer, INL inner nuclear layer, IPL inner plexiform layer, ONL outer nuclear layer, OPL outer plexiform layer. Scale bar: $50 \mu \mathrm{m}$ (a, c, p) 